\title{
Current State-of-the-Art Therapy for Advanced Squamous Cell Lung Cancer
}

\author{
Giorgio V. Scagliotti, MD, PhD, Silvia Novello, MD, PhD, Simonetta Rapetti, MD, and Mauro Papotti, MD
}

\section{OVERVIEW}

Squamous cell carcinoma (SCC) represents the second most common histologic subtype of lung cancer (preceded only by adenocarcinoma). SSC of the lung is prevalently diagnosed in smokers and has been described as a preferentially centrally located tumor in which the main airways are commonly involved. Clinically, it presents with predominant locoregional signs and symptoms, but in recent years an increasing frequency of peripheral SCC of the lung has been reported. Pathologic diagnosis can be easily made through light microscopy and immunohistochemistry. The treatment approach for early-stage disease does not differ from that of other histologic subtypes of non-small cell lung cancer; in locally advanced unresectable or metastatic disease, doublet chemotherapy regimens (including cisplatin or carboplatin and a third-generation agent such as gemcitabine, taxanes, or vinorelbine) remain the cornerstone of front-line systemic treatment. Conversely, a single agent, mainly docetaxel, is the preferred treatment in second-line treatment. In unselected patient populations, targeted therapies have been extensively tested in combination with cytotoxic chemotherapy with disappointing results because of increased toxicity or lack of improvement in efficacy outcomes. Genomic alterations in SCC of the lung have not been comprehensively characterized, and no molecularly targeted therapies have been specifically developed for the treatment of this disease, but recently immune checkpoints have emerged as new therapeutic agent.

$\mathrm{L}$ ung cancer is the leading cause of cancer-related death worldwide, with 1.4 million deaths estimated in $2010 .^{1}$ Approximately $85 \%$ of the newly diagnosed lung cancers are non-small cell lung cancer (NSCLC) and, among these, $20 \%$ to $30 \%$ are squamous cell carcinoma (SCC). ${ }^{2}$ SCC of the lung is strongly associated with cigarette smoking and was, in the past, the most common histologic subtype of lung cancer. Its incidence has now been surpassed by adenocarcinoma, reflecting trends in reduced tobacco exposure, with the introduction of filtered and low-tar cigarettes, as well as changes in cigarette smoke inhalation patterns; nevertheless, SCC of the lung is still responsible for 40,000 deaths annually in the United States. ${ }^{3}$

SCC is a preferentially centrally located tumor in which the main airways (trachea, carina, lobar, and segmental bronchi) are commonly involved, which is responsible for symptoms and signs such as cough, dyspnea, atelectasis, and obstructive pneumonia, as well as hemoptysis. In recent years, an increasing frequency of peripheral SCC of the lung has been reported. Central tumor necrosis with or without cavitation is a common radiological finding. SCC of the lung is the most common histotype associated with Pancoast syndrome.

\section{PATHOLOGY}

SCC arises from dysplastic and metaplastic squamous epithelium, and, in addition to the classic SCC, the World
Health Organization recognizes several additional histologic patterns including papillary, clear cell, small cell, and basaloid variants. ${ }^{4}$ In histologic specimens, SCC is defined by keratinization and/or intercellular bridges. In smears and liquid-based preparations of well-differentiated SCC, intact intercellular bridges and keratin pearls are uncommon, ${ }^{5}$ but they may be readily seen in cell block preparations. Rather, squamous cells are characterized by dense dark blue cytoplasm or orangeophilia on Diff-Quik and Papanicolaoustained slides, respectively. As SCC becomes less differentiated, the aforementioned features become less prominent. Poorly differentiated carcinomas form syncytial groups, especially in aspirates, and the cells have cyanophilic cytoplasm, higher nuclear/cytoplasmic ratios, and prominent nucleoli. ${ }^{5}$

Most SCCs express high molecular-weight cytokeratin, cytokeratins 5/6 (CK5/6), and p63. Thyroid transcription factor-1 (TTF-1) and cytokeratin 7 (CK7) are present in a subset of cases, possibly as a result of mixed adeno-squamous phenotypes or non-specific staining. p63 has emerged as the "front-runner" of the squamous markers. ${ }^{6}$ The perplexing issue with p63 is its unexpected occasional presence in various other tumor types, such as lung adenocarcinomas and lymphomas, as well as some sarcomas and various cancers (e.g., ovarian, endometrial, breast, and colorectal).

From the Department of Oncology, University of Torino, San Luigi Hospital, Orbassano, Torino, Italy.

Authors' disclosures of potential conflicts of interest are found at the end of this article.

Corresponding author: Giorgio V. Scagliotti, MD, PhD, Department of Oncology, University of Torino, San Luigi Hospital, Regione Gonzole 10, 10043 Orbassano (Torino), Italy, 414, 616; email: giorgio.scagliotti@unito.it.

(c) 2013 by American Society of Clinical Oncology. 
p40 is a relatively unknown antibody that recognizes $\Delta$ Np63-a p63 isoform and was found to be highly specific for squamous/basal cells. A large retrospective study compared the standard p63 antibody (4A4) and p40 in a series of 470 tumors, which included lung SCCs (81 patients), adenocarcinomas (237 patients), and large cell lymphomas (152 patients). p63 was positive in $100 \%$ of SCCs, $31 \%$ of adenocarcinomas, and $54 \%$ of large cell lymphomas (sensitivity $100 \%$, specificity $60 \%$ ). In contrast, although p40 was also positive in $100 \%$ of SCCs, only $3 \%$ of adenocarcinomas and none of large cell lymphomas had p40 labeling (sensitivity $100 \%$, specificity $98 \%$ ). In summary, p40 is equivalent to p63 in sensitivity for SCC, but it is markedly superior to p63 in specificity, which eliminates a potential pitfall of misinterpreting a p63-positive adenocarcinoma or unsuspected lymphoma as a SCC. 6

In the daily clinical practice, lung cancer immunohistochemical characterization may be of additional value in those $20 \%$ to $30 \%$ of poorly differentiated NSCLC cases which do not show a clear-cut differentiation at light microscope. A panel of immunohistochemical markers highlighting cell differentiation lineages may be especially useful when the scant diagnostic material does not allow a definitive subtyping. In a retrospective study, it has been shown that a fourantibody panel approach, including CK7, CK5/6, TTF1, and p63 (with the additional use of p40, napsin $\mathrm{A}$, and desmocollin-3 in discordant cases), contributed to refine lung cancer classification in fine needle aspiration cytology cell blocks, remarkably reducing the NSCLC-not otherwise specified diagnostic category. ${ }^{7}$

\section{KEY POINTS}

Squamous cell carcinoma (SCC) of the lung is strongly associated with cigarette smoking. Its incidence has now been surpassed by adenocarcinoma, reflecting trends in reduced tobacco exposure.

- On histologic sections, SCC is defined by keratinization and/or intercellular bridges. By immunohistochemistry, it express high molecular-weight cytokeratin, cytokeratins $5 / 6, p 63$, and $p 40$. Thyroid transcription factor- 1 and cytokeratin 7 staining are present in a subset of cases.

- For patients with locally advanced or metastatic SCC of the lung, two-drug chemotherapy regimens (including a platinum agent and a third-generation agent such as gemcitabine, taxanes, or vinorelbine) remain the cornerstone of treatment options.

- To enhance chemotherapy response, the DNA damage response may be targeted by reactivation of $\mathrm{p} 53$, by inhibition of cell cycle checkpoints or by inhibition of DNA repair processes, but none of these approaches have been clinically validated at this time.

- In SCC there is no established role for targeted therapies used either alone or in combination with chemotherapy.
This cheap analysis can overlap the results obtained with more expensive (\$8 to $\$ 10$ compared with hundreds of U.S. dollars) and time-consuming (one day compared with several days) molecular techniques, such as the investigation of specific microRNAs, as proposed by Lebanony and colleagues. $^{8}$

\section{CURRENT TREATMENT AND OUTCOME Front-Line Management}

The discovery of recurrent mutations in the epidermal growth factor receptor (EGFR) kinase, as well as fusions involving anaplastic lymphoma kinase $(A L K)$, has led to a marked change in the treatment of patients with lung adenocarcinoma. More recent data have suggested that targeting mutations in BRAF, AKT1, ERBB2, and PIK3CA and fusions that involve ROS1 and RET may also be successful. ${ }^{9}$ Unfortunately, activating mutations in EGFR and ALK fusions are typically absent in lung SCC, ${ }^{10}$ and targeted agents developed for lung adenocarcinoma are largely ineffective against lung SCC. The occasional detection of these mutations in samples diagnosed as SCC is a result of challenges with the diagnosis of adeno-squamous carcinoma and adenocarcinoma, which can be largely resolved by comprehensive pathologic assessment incorporating immunohistochemical markers. ${ }^{10}$

Historically, approaches to the treatment of NSCLC were uniform, and histologic subtypes within NSCLC did not significantly affect treatment decisions. ${ }^{11}$ Retrospective investigation of some of the phase III studies investigating the role of modern platinum-based doublets failed to detect a differential activity of one specific doublet according to histology, and in one study squamous histology was associated with better outcomes. ${ }^{12}$

For patients with locally advanced or metastatic SCC of the lung, two-drug chemotherapy regimens (including a platinum agent and a third-generation agent such as gemcitabine, taxanes, or vinorelbine) currently remain the cornerstone of treatment options. ${ }^{13}$ However, outcomes associated with conventional therapies remain poor, underlining the clear need for alternative approaches to treatment in this patient population. Several studies ${ }^{14}$ have shown an inferior activity of pemetrexed in combination with cisplatin in SCC of the lung, and this agent is not currently licensed for the treatment of this tumor type. These results may be related to a different expression of thymidylate synthase (TS), one of the main enzymes of the folate pathway targeted by pemetrexed, in different histologic subtypes of NSCLC, leading to sensitivity to pemetrexed which was lower in the SCC and higher in the adenocarcinoma of the lung and prospective studies to validate this hypothesis are currently underway. ${ }^{15}$

Recently, in a large phase III trial (1,052 patients), the efficacy and safety of albumin-bound paclitaxel (nab-paclitaxel [nab-PC]) plus carboplatin was compared with solventbased paclitaxel (sb-PC) plus carboplatin in advanced NSCLC; objective response rate (ORR) was the primary outcome. nab-PC demonstrated a significantly higher ORR than 
sb-PC (33\% vs. $25 \%$; $\mathrm{p}=0.005)$ and in patients with squamous histology ( $41 \%$ vs. $24 \%$; $p<0.001$ ). nab-PC was as effective as sb-PC in patients with nonsquamous histology ( $26 \%$ vs. $25 \%$; $\mathrm{p}=0.808$ ). This improvement in ORR unfortunately did not translate into any meaningful progressionfree and overall survival benefit. ${ }^{16}$ This differential activity might be partially attributable to aberrant caveolin-1 expression in SCC resulting in an higher intratumoral accumulation. ${ }^{16}$ The expression of secreted protein acidic and rich in cysteine (SPARC), which facilitates the accumulation of albumin in the tumor and thus increases intracellular concentrations of the cytotoxic agent, has also been suggested. SPARC expression has been linked to a higher response rate in patients with head and neck cancers, which are predominantly of squamous histology. ${ }^{17}$

To enhance chemotherapy response, the DNA damage response may be targeted by reactivation of p53, by inhibition of cell cycle checkpoints, or by inhibition of DNA repair processes. ${ }^{18}$ Platinum resistance, either intrinsic or acquired, is a major clinical problem, as additional agents that can be added to therapy in order to circumvent tumor resistance do not currently exist. Platinum chemotherapy has been tested with poly(ADP-ribose) polymerase (PARP) inhibition in clinical trials. The rationale for the combination is based on preclinical observations that PARP inhibitors preferentially kill neoplastic cells and induce complete or partial regression of a wide variety of human tumor xenografts in nude mice treated with platinum chemotherapy. ${ }^{19}$ However, the full function of PARP in DNA repair is not clear ${ }^{20}$ and, as a result, the biologic mechanisms of chemosensitization of cancer cells to platinum chemotherapy by PARP inhibition remain to be resolved. Iniparib, initially developed as a PARP inhibitor, has a different mechanism of action from other PARP inhibitors, because it forms a covalent bond. Additional preclinical studies have shown that it induces g-H2AX marker of DNA damage, does not inhibit PARP1/2 at physiologic drug concentrations, and potentiates cell cycle arrest induced by DNA-damaging agents. ${ }^{21}$ This agent already entered phase III clinical trials for breast cancer and NSCLC. The ECLIPSE study, a phase III trial for patients with advanced squamous cell lung cancer receiving a combination of carboplatin plus gemcitabine either with or without iniparib, has recently completed accrual, but data are not yet available. A previous phase II study conducted in all NSCLC histologies failed to show an improvement in response rate. ${ }^{21}$ Relatively little is known about PARP1 protein levels and activity in normal cells compared with tumor cells and how this contributes to a therapeutic index when PARP inhibitors are used in conjunction with DNA-damaging agents.

Histone deacetylases (HDACs) may also represent druggable targets for the inhibition of DNA repair, as several class I and class II HDACs (HDAC1-4) have been shown to promote DNA repair. A phase II study has been conducted in NSCLC showing hints of activity. ${ }^{22}$ However, most current inhibitors of epigenetic modifiers lack target specificity, and genome-wide alterations may result in adverse effects on the tumors or increase side effects when combined with chemotherapy. A better insight into the mechanisms through which these compounds sensitize to chemotherapy may lead to the identification of relevant target genes and ultimately yield more selective therapeutic strategies. ${ }^{18}$

Some studies have evaluated combinations of targeted therapies plus standard-of-care chemotherapy. Increased risk of bleeding has been reported in some, but not all, studies evaluating vascular endothelial growth factor (VEGF) pathway inhibitors as first-line therapy for advanced NSCLC in patient populations that included squamous histology. In a phase II study, four of 13 patients with squamous NSCLC and two patients with nonsquamous histology who received bevacizumab plus carboplatin and paclitaxel had severe lifethreatening hemorrhagic events, ${ }^{23}$ and this clinical toxicity led to the exclusion of patients with squamous histology from the phase III studies investigating bevacizumab in NSCLC. More recently, in a small phase II study performed in $31 \mathrm{pa}$ tients with metastatic SCC of the lung, the rate of pulmonary hemorrhage was low, but it was correctly concluded that treatment of SCC of the lung with bevacizumab should be considered experimental. ${ }^{24}$

Several multitargeted, antiangiogenic agents have been studied in clinical trials for patients with advanced NSCLC. Most of these agents inhibit the VEGF receptor (VEGFR), and some also inhibit platelet-derived growth factor receptor (PDGFR), BRAF, and EGFR. In a phase III study of carboplatin plus paclitaxel with and without sorafenib in first-line advanced NSCLC, the primary endpoint of overall survival (OS) was not met and the study was terminated early because of the detrimental effect of sorafenib on patients with SCC and lack of effect in the population with nonsquamous cell carcinoma. ${ }^{25}$ Differently from what has been previously observed with bevacizumab, in this study the life threatening toxicities were multifactorial. Motesanib is a VEGFR-1, -2, and -3, PDGFR, and KIT inhibitor that is currently in clinical development in multiple tumor types, including NSCLC. MONET-1 was another phase III trial investigating chemotherapy with or without motesanib (125 mg once daily); it was temporarily suspended because of an increased risk of hemoptysis in patients with squamous cell histology. These patients discontinued the trial, the study subsequently restarted, and more than 1,000 patients with nonsquamous cell histology were treated, but unfortunately the final efficacy analysis did not show any meaningful benefit. ${ }^{26}$

A great deal of clinical interest was spurred by the early results from a randomized phase II study of chemotherapy with or without figitumumab, an antibody against insulinlike growth factor type 1 receptor, which showed promising activity in phase II studies, especially among patients with squamous cell lung cancer, ${ }^{27}$ but unfortunately these data are currently being reassessed because of an inaccurate data analysis.

At present, only one study that enrolled patients with squamous histology as part of a larger study in advanced NSCLC (377 of 1,125 patients; $34 \%$ ) demonstrated an OS benefit. 
The FLEX study investigated the addition of cetuximab to cisplatin plus vinorelbine and showed an improved OS of 1.2 months compared with chemotherapy alone in an openlabel, randomized phase III trial in patients with NSCLC. When patients were stratified by EGFR expression according to a semi-quantitative score, a higher percentage of SCCs of the lung was seen in the high EGFR expression group. For patients in the high EGFR expression group, OS was 2.4 month longer in the chemotherapy plus cetuximab arm (hazard ratio, $\mathrm{HR}=0.73, \mathrm{p}=0.011$ ) while no corresponding benefit was seen in the low EGFR expression group. The subgroup of patients with SCC and high EGFR score showed the higher benefit $(\mathrm{HR}=0.62) .{ }^{28}$ Although some therapeutic guidelines recommend the addition of cetuximab to chemotherapy for the management of metastatic NSCLC ${ }^{29}$ the agent is not currently approved in Europe and in the United States for this specific indication.

Immune checkpoints have emerged as new therapeutic targets. Blocking inhibitory pathways via monoclonal antibodies, such as the anti-cytotoxic T-lymphocyte antigen-4 antibody, anti-programmed cell death-1 antibody, and antiprogrammed cell death-1 ligand antibody, has the ability to break down the shield that tumors co-opt for their defense.

In a phase II study ipilumumab, an anti-cytotoxic T-cell lymphocyte-4 monoclonal antibody, when administered with carboplatin and paclitaxel according to a phased regimen (two doses of placebo plus carboplatin and paclitaxel followed by 4 doses of ipilumumab plus carboplatin and paclitaxel then followed by a maintenance phase with ipilumumab alone) showed a superior activity in $\mathrm{SCC}^{30}$. A phase III study in SCC is currently ongoing.

\section{SECOND-LINE THERAPY FOR ADVANCED NSCLC}

The availability of tolerable and active therapeutic agents for second- or third-line treatment also contributed to the improved survival outcome noted with NSCLC in the last decade. Randomized controlled trials have established the efficacy of pemetrexed, docetaxel, and erlotinib as salvage treatment agents for patients with NSCLC whose disease progressed after front-line therapy. Apart from the restriction of pemetrexed to patients with tumors with a nonsquamous histology, the choice between these agents in the salvage setting is driven primarily by drug toxicity profile and patient choices. It is, however, conceivable that the selection of EGFR kinase inhibitors will require the demonstrable presence of activating EGFR mutations, similar to the practice standard in the front-line setting. ${ }^{31} \mathrm{Al}$ though maintenance and second/third line phase III studies with erlotinib 32,33 showed survival improvement in the overall treated population the benefit was largely driven by EGFR mutational status which is definitively less frequently detected in SCC.

However due to limited current treatment options an ongoing phase III trial (LUX LUNG 8) compare the efficacy of afatinib (BIBW 2992) versus erlotinib as second line treatment in patients with SCC of the lung, as measured by progression-free survival(ClinicalTrials.gov Identifier: NCT01523587).

A phase I trial of the anti-PD-1 antibody (BMS-936558) administered once every 2 weeks included a large expansion cohort of patients with NSCLC ${ }^{34}$. Patients with advanced NSCLC who had been previously treated with a prior platinum-containing regimen were eligible but could have received no more than five prior treatment regimens for their advanced disease. Besides being included in the doseescalation portion of the trial, patients with NSCLC were randomly assigned to 1,3 , and $10 \mathrm{mg} / \mathrm{kg}$ to include equal numbers of squamous and non-squamous cell histology at each dose level. Of the 76 patients with NSCLC evaluable for response, 14 patients had objective response (response rate, $18 \%)$. An unprecedented response rate of $33 \%$ was observed among the 18 patients with SCC. Phase III studies are being planned in the second-line treatment setting in advanced NSCLC. Other anti PD-1 and antiPDL-1 antibodies are currently being evaluated in phase I clinical trials.

\section{CONCLUSION}

SCC is a histologic subtype of lung cancer, easily diagnosed through light microscopy or immunohistochemistry and highly tobacco-related, which has a higher mutation rate compared with lung adenocarcinoma. As opposed to adenocarcinoma, no established driver mutation has been definitely proven and no specific targeted therapy is currently available. Doublet chemotherapy regimens (including cisplatin or carboplatin and a third-generation agent such as gemcitabine, taxanes, or vinorelbine) remain the cornerstone of the front-line systemic treatment, while a single agent, mainly docetaxel, is the preferred treatment in the secondline setting.

\section{Disclosures of Potential Conflicts of Interest}

Relationships are considered self-held and compensated unless otherwise noted. Relationships marked "L" indicate leadership positions. Relationships marked "I" are those held by an immediate family member; those marked "B" are held by the author and an immediate family member. Relationships marked "U" are uncompensated. 


\section{References}

1. World Health Organization. Cancer, fact sheet no. 297 http:// www.who.int/mediacentre/factsheets/fs297/en. Accessed September 2012.

2. Travis WD. Pathology of lung cancer. Clin Chest Med. 2011;32:669-692.

3. Siegel R, Naishadham D, Jemal A. Cancer statistics, 2012. CA Cancer J Clin. 2012;62:10-29.

4. Travis WD, Brambilla E, Muller-Hermelink HK, et al (eds). World Health Organization Classification of Tumors. Pathology and Genetics of Tumours of the Lung, Pleura, Thymus, and Heart. Lyon, France: IARC Press, 2004;26-30.

5. Johnston WW, Elson CE. Respiratory tract. In Bibbo M (ed). Comprehensive Cytopathology. 2nd ed. Philadelphia, PA: W.B. Saunders Company, 1997;325-401.

6. Bishop JA, Teruya-Feldstein J, Westra WH, et al. p40 ( $\Delta$ Np63) is superior to $\mathrm{p} 63$ for the diagnosis of pulmonary squamous cell carcinoma. Mod Pathol. 2012;25:405-415.

7. Righi L, Graziano P, Fornari A, et al. Immunohistochemical subtyping of nonsmall cell lung cancer not otherwise specified in fine-needle aspiration cytology: a retrospective study of 103 cases with surgical correlation. Cancer. 2011;117:3416-3423.

8. Lebanony D, Benjamin H, Gilad S, et al. Diagnostic assay based on hsamiR-205 expression distinguishes squamous from nonsquamous nonsmall-cell lung carcinoma. J Clin Oncol. 2009;27:2030-2037.

9. Pietanza MC, Ladanyi M. Bringing the genomic landscape of small-cell lung cancer into focus. Nature Genetics. 2012;44:1074-1075.

10. Rekhtman N, Paik PK, Arcila ME, et al. Clarifying the spectrum of driver oncogene mutations in biomarker-verified squamous carcinoma of lung: lack of EGFR/KRAS and presence of PIK3CA/AKT1 mutations. Clin Cancer Res. 2012;18:1167-1176.

11. Hirsch FR, Spreafico A, Novello S, et al. The prognostic and predictive role of histology in advanced non-small cell lung cancer: a literature review. J Thorac Oncol. 2008;3:1468-1481.

12. Scagliotti GV, De Marinis F, Rinaldi M, et al. The role of histology with common first-line regimens for advanced non-small cell lung cancer: a brief report of the retrospective analysis of a three-arm randomized trial. J Thorac Oncol. 2009;4:1568-1571.

13. Peters $S$, Adjei AA, Gridelli C, et al. Metastatic non-small-cell lung cancer (NSCLC): ESMO Clinical Practice Guidelines for diagnosis, treatment and follow-up. Ann Oncol. 2012;23 (suppl 7):vii56-vii64.

14. Scagliotti G, Brodowicz T, Shepherd FA, et al. Treatment-by-histology interaction analyses in three phase III trials show superiority of pemetrexed in nonsquamous non-small cell lung cancer. J Thorac Oncol. 2011;6:64-70.

15. Ceppi P, Volante M, Saviozzi S, et al. Squamous cell carcinoma of the lung compared with other histotypes shows higher messenger RNA and protein levels for thymidylate synthase. Cancer. 2006;107:1589-1596.

16. Socinski MA, Bondarenko I, Karaseva NA, et al. Weekly nab-paclitaxel in combination with carboplatin versus solvent-based paclitaxel plus carboplatin as first-line therapy in patients with advanced non-smallcell lung cancer: final results of a phase III trial. J Clin Oncol. 2012;30: 2055-2062.

17. Desai N, Trieu V, Damascelli B, et al. SPARC expression correlates with tumor response to albumin-bound paclitaxel in head and neck cancer patients. Transl Oncol. 2009;2:59-64.

18. Bouwman P, Jonkers J. The effects of deregulated DNA damage signalling on cancer chemotherapy response and resistance. Nat Rev Cancer. 2012;12:587-598.

19. Miknyoczki, SJ, Jones-Bolin S, Pritchard S et al. Chemopotentiation of temozolomide, irinotecan, and cisplatin activity by CEP-6800, a poly(ADP-ribose) polymerase inhibitor. Mol Cancer Ther. 2003;2:371382.

20. Ahel I, Ahel D, Matsusaka T, et al. Poly(ADP-ribose)-binding zinc finger motifs in DNA repair/checkpoint proteins. Nature. 2008;451:81-85.

21. Novello S, Felip E, Barlesi F, et al. Results of a phase 2 study of gemcitabine/cisplatin/iniparib (GCI) versus gemcitabine/cisplatin (GC) in patients with advanced NSCLC. Eur J Cancer. 2011;47 (suppl 1; abstr 9006):S593.

22. Ramalingam SS, Maitland ML, Frankel P, et al. Carboplatin and paclitaxel in combination with either vorinostat or placebo for first-line therapy of advanced non-small-cell lung cancer. JClin Oncol. 2010;28:56-62.

23. Johnson DH, Fehrenbacher L, Novotny WF, et al. Randomized phase II trial comparing bevacizumab plus carboplatin and paclitaxel with carboplatin and paclitaxel alone in previously untreated locally advanced or metastatic non-small-cell lung cancer. J Clin Oncol. 2004;22:21842191.

24. Hainsworth JD, Fang L, Huang JE, et al. BRIDGE: an open-label phase II trial evaluating the safety of bevacizumab + carboplatin/paclitaxel as first-line treatment for patients with advanced, previously untreated, squamous non-small cell lung cancer. J Thorac Oncol. 2011;6:109-114.

25. Scagliotti G, Novello S, von Pawel J, et al. Phase III study of carboplatin and paclitaxel alone or with sorafenib in advanced non-small-cell lung cancer. J Clin Oncol. 2010;28:1835-1842.

26. Scagliotti GV, Vynnychenko I, Park K, et al. International, randomized, placebo-controlled, double-blind phase III study of motesanib plus carboplatin/paclitaxel in patients with advanced non-squamous nonsmall-cell lung cancer: MONET1. J Clin Oncol. 2012;30:2829-2836.

27. Karp DD, Paz-Ares LG, Novello S, et al. Phase II study of the antiinsulin-like growth factor type 1 receptor antibody CP-751,871 in combination with paclitaxel and carboplatin in previously untreated, locally advanced, or metastatic non-small-cell lung cancer. J Clin Oncol. 2009; 27:2516-2522. Retraction in: J Clin Oncol. 2012;30:4179.

28. Pirker R, Pereira JR, von Pawel J, et al. EGFR expression as a predictor of survival for first-line chemotherapy plus cetuximab in patients with advanced non-small-cell lung cancer: analysis of data from the phase 3 FLEX study. Lancet Oncol. 2012;13:33-42.

29. National Comprehensive Cancer Network. NCCN Clinical Practice Guidelines in Oncology Non-Small Cell Lung Cancer. Published February 2013. Accessed March 13, 2013.

30. Lynch TJ, Bondarenko I, Luft A, et al. Ipilumumab in combination with paclitaxel and carboplatin as first-line treatment in stage IIIB/IV nonsmall cell lung cancer: results from a randomized, double-blind, multicenter phase II study. J Clin Oncol. 2012;30:2046-2054.

31. Ramalingam SS, Owonikoko TK, Khuri FR. Lung cancer: New biological insights and recent therapeutic advances. CA Cancer J Clin. 2011;61: 91-112.

32. Shepherd FA, Rodrigues Pereira J, Ciuleanu T, et al. Erlotinib in previously treated non-small cell lung cancer. N Engl J Med. 2005;353:123132.

33. Cappuzzo F, Ciuleanu T, Stelmakh L, et al. Erlotinib as maintenance treatment in advanced non-small-cell lung cancer: a multicentre, randomised, placebo-controlled phase 3 study. Lancet Oncol. 2010;11:521529.

34. Topalian SL, Hodi FS, Brahmer JR, et al. Safety, activity, and immune correlates of anti-PD-1 antibody in cancer. N Engl J Med. 2012;266: 2443-2454. 